\title{
Perfil epidemiológico, hematológico e bioquímico em cães com Dirofilaria sp. no
}

\section{Ceará}

\author{
Epidemiological, hematological and biochemical profile in dogs with Dirofilaria sp. in Ceara \\ Perfil epidemiológico, hematológico y bioquímico en perros con Dirofilaria sp. en Ceara
}

Recebido:16/06/2021 | Revisado: 24/06/2021 | Aceito: 26/06/2021 | Publicado: 11/07/2021

Lorena Santos Bezerra

ORCID: https://orcid.org/0000-0001-9637-6528

Universidade de Fortaleza, Brasil

E-mail: lorenasantos.medvet@ gmail.com

Glenda Roberta Freire Lima

ORCID: https://orcid.org/0000-0002-3867-7389 Universidade Estadual do Ceará, Brasil

E-mail: glendaroberta.medvet@gmail.com

Vitória Maria Jorge de Araújo

ORCID: https://orcid.org/0000-0001-6489-6959

Universidade Estadual do Ceará, Brasil

E-mail: vitoria.m.arauj@gmail.com

Germano Gonçalves Teixeira

ORCID: https://orcid.org/0000-0002-6586-1819

Universidade Estadual do Ceará, Brasil

E-mail: germanoggt06@gmail.com

João Marcelo Alves Coelho

ORCID: https://orcid.org/0000-0001-6289-5741

Faculdade CISNE, Brasil

E-mail: marcello.alves1254@gmail.com

Flavia de Azevedo Farzat

ORCID: https://orcid.org/0000-0003-2849-2695

Universidade Estadual do Ceará, Brasil

E-mail: flaviafarzat@hotmail.com

Elana da Silva Oliveira

ORCID: https://orcid.org/0000-0002-5121-4389

Universidade Estadual do Ceará, Brasil E-mail: professoraelana@gmail.com

Vitória Costa Pinheiro

ORCID: https://orcid.org/0000-0001-5865-3053 Faculdade CISNE, Brasil

E-mail: vitoria00costa2016@hotmail.com

Adriane Luzia Da Silva Mendes

ORCID: https://orcid.org/0000-0001-8402-8833 Faculdade CISNE, Brasi

E-mail: adrianelsm17@gmail.com

Paola Ramires

ORCID: https://orcid.org/0000-0002-6061-2872 Universidade Estadual do Ceará, Brasil

E-mail: paolaramiress@gmail.com

Isaac Neto Goes da Silva

ORCID: https://orcid.org/0000-0002-6055-1790 Universidade Estadual do Ceará, Brasil

E-mail: isaac.neto@uece.br

Breno Queiroz Pinheiro

ORCID: https://orcid.org/0000-0001-6363-6952 Universidade Estadual do Ceará, Brasil

E-mail: breno.queiroz@uece.br

Victor Hugo Vieira Rodrigues

ORCID: https://orcid.org/0000-0003-2974-8015

Centro Universitário Maurício de Nassau, Brasil

E-mail: victorvieira81@gmail.com

\section{Resumo}

A dirofilariose é uma importante zoonose considerada endêmica no Brasil, cuja transmissão acontece principalmente por meio de mosquitos vetores dos gêneros Culex, Aedes e Anopheles e que tem o cão doméstico como hospedeiro. 
Apesar da maioria dos animais serem assintomáticos, a doença pode afetar diversos órgãos, culminando em possíveis alterações em diagnósticos laboratoriais. Pode ser empregado diversos diagnósticos, sendo a visualização de microfilárias de Dirofilaria spp. em esfregaço sanguíneo o mais utilizado. O objetivo do trabalho foi verificar o perfil hematológico e bioquímico de cães diagnosticados com microfilárias de Dirofilaria immitis na cidade de Fortaleza, Ceará. Foram selecionadas amostras sanguíneas no período de agosto de 2014 a junho de 2018. Nas amostras em que houve positividade para a presença de microfilárias no esfregaço sanguíneo foi realizado um levantamento das alterações encontradas nos exames hematológicos e bioquímicos. Em 2.400 amostras de sangue analisadas, 26 apresentaram microfilárias $(1,1 \%)$, com $46 \%$ de anemia e $46 \%$ com sinais de regeneração eritrocitária, $42 \%$ apresentaram trombocitopenia e $46 \%$ hiperproteinemia, com linfopenia em $61 \%$ dos casos. No perfil bioquímico, foram observadas discretas alterações abaixo do normal, exceto para albumina, que foi reduzida em $62 \%$ dos casos. A maioria dos analitos apresentaram valores normais, indicando que não havia uma hiperfunção ou hiperatividade dos órgãos. Logo, a dirofilariose influenciou na imunologia canina e, provavelmente, induziu a uma redução no número de plaquetas e aumento nas proteínas totais pelo estímulo inflamatório gerado.

Palavras-chave: Dirofilariose; Hematologia; Marcadores bioquímicos; Cães.

\begin{abstract}
Heartworm disease is an important zoonosis considered to be endemic in Brazil, whose transmission occurs mainly through mosquito vectors of the genera Culex, Aedes and Anopheles and which have the domestic dog as its host. Although most animals are asymptomatic, the disease can affect several organs, culminating in possible changes in laboratory diagnoses. Different diagnoses can be used, being the visualization of microfilariae of Dirofilaria spp. in blood smear the most used. The objective of this work was to verify the hematological and biochemical profile of dogs diagnosed with Dirofilaria immitis microfilariae in the city of Fortaleza, Ceará. Blood samples were selected from August 2014 to June 2018. In samples where there was positivity for the presence of microfilariae in the blood smear, a survey of the changes found in hematological and biochemical tests was carried out. In 2,400 blood samples analyzed, 26 had microfilariae $(1.1 \%)$, with $46 \%$ of anemia and $46 \%$ with signs of erythrocyte regeneration, $42 \%$ had thrombocytopenia and $46 \%$ hyperproteinemia, with lymphopenia in $61 \%$ of cases. In the biochemical profile, slight alterations below normal were observed, except for albumin, which was reduced in $62 \%$ of cases. Most analytes had normal values, indicating that there was no organ hyperfunction or hyperactivity. Therefore, heartworm disease influenced canine immunology and probably induced a reduction in the number of platelets and an increase in total proteins by the inflammatory stimulus generated.
\end{abstract}

Keywords: Heartworm disease; Hematology; Biochemical markers; Dogs.

\title{
Resumen
}

El gusano del corazón es una zoonosis importante considerada endémica en Brasil, cuya transmisión ocurre principalmente a través de mosquitos vectores de los géneros Culex, Aedes y Anopheles y que tienen al perro doméstico como huésped. Aunque la mayoría de los animales son asintomáticos, la enfermedad puede afectar a varios órganos, culminando en posibles cambios en los diagnósticos de laboratorio. Se pueden utilizar varios diagnósticos, siendo la visualización de microfilarias de Dirofilaria spp. en frotis de sangre el más utilizado. El objetivo de este trabajo fue verificar el perfil hematológico y bioquímico de perros diagnosticados con microfilarias de Dirofilaria immitis en la ciudad de Fortaleza, Ceará. Se seleccionaron muestras de sangre desde agosto de 2014 hasta junio de 2018. En las muestras donde hubo positividad para la presencia de microfilarias en el frotis de sangre, se realizó un relevamiento de los cambios encontrados en las pruebas hematológicas y bioquímicas. En 2.400 muestras de sangre analizadas, 26 presentaban microfilarias $(1,1 \%)$, con $46 \%$ de anemia y $46 \%$ con signos de regeneración eritrocitaria, $42 \%$ trombocitopenia y $46 \%$ hiperproteinemia, con linfopenia en $61 \%$ de los casos. En el perfil bioquímico se observaron ligeras alteraciones por debajo de lo normal, a excepción de la albúmina, que se redujo en el $62 \%$ de los casos. La mayoría de los analitos tenían valores normales, lo que indica que no había hiperfunción o hiperactividad de órganos. Por tanto, la enfermedad del gusano del corazón influyó en la inmunología canina y probablemente indujo una reducción del número de plaquetas y un aumento de las proteínas totales por el estímulo inflamatorio generado.

Palabras clave: Dirofilariosis; Hematología; Marcadores bioquímicos; Perro.

\section{Introdução}

A dirofilariose é uma zoonose reemergente causada pelo helminto Dirofilaria immitis (Silva \& Langoni, 2009). Os nematóides fêmeas liberam sangue circulante com larvas (microfilárias) que se tornam infectantes após serem ingeridas por mosquitos dos gêneros Culex, Aedes e Anopheles que atuam como hospedeiro intermediário e vetor (Mircean et al, 2017). Apresenta ampla distribuição geográfica, sendo considerada endêmica no Brasil, com elevada prevalência de cães microfilarêmicos (Silva \& Langoni, 2009). No entanto apresenta maior prevalência em regiões litorâneas (Labarthe et al., 2014) com clima tropical ou subtropical (Moreira et al., 2019), sendo o Nordeste indicado como região com maior prevalência 
média se comparado a regiões Sudeste e Sul do Brasil (Labarthe et al., 2014; Figueredo et al., 2017).

Os fatores de riscos para dirofilariose, até então estudados, trazem evidências de que cães mais velhos e semidomiciliados apresentam uma prevalência significativa de dirofilariose (Wang et al., 2016; Calazans, 2018), cães de grande porte, de raças mestiças e que realizam atividades ao ar livre como guarda e caça também são considerados como grupo de risco para a infecção (Vieira et al., 2014), no entanto não há relatos de distinção de sexo na prevalência de dirofilariose (Wang et al., 2016). Um fator importante para a prevenção da doença, são os cães dormirem dentro de casa, para evitar exposição dos animais aos vetores, diminuindo o risco de infecção. (Calazans, 2018).

A doença pode afetar diversos órgãos, incluindo o coração, os pulmões, fígado e rins (Hodges \& Rishniw, 2008), no qual o estado clínico está relacionado ao número de parasitos adultos presentes e a resposta de cada animal à infecção (DantasTorres \& Otranto, 2020). Em animais sintomáticos, destaca-se a insuficiência cardiorrespiratória (Smith Jr. et al., 2015). O diagnóstico da doença é realizado a partir de testes imunoenzimáticos, provas moleculares ou na demonstração das microfilárias na circulação, tais como o esfregaço sanguíneo, teste de Knott, ou gota espessa (Furtado et al., 2009; Silva \& Langoni, 2009; Magnis et al., 2013), sendo a técnica de Knott modificada a de maior sensibilidade (Silva \& Langoni, 2009).

Considerando os fatores ambientais, como o clima tropical da região Nordeste e características da população canina favoráveis a prevalência de dirofilariose, o objetivo deste estudo foi investigar alterações hematológicas e bioquímicas através de hemogramas em cães que apresentaram microfilárias de Dilofilaria spp., em sangue periférico, analisadas por meio de esfregaços sanguíneos, e, com isso realizar levantamento de dados, que se deram em 5 anos, no município de Fortaleza, Ceará.

\section{Metodologia}

A pesquisa foi realizada no Laboratório de Patologia Clínica Veterinária da Faculdade de Veterinária da Universidade Estadual do Ceará (FAVET/UECE) localizado na cidade de Fortaleza, Ceará. As amostras foram selecionadas casualmente de cães provenientes da rotina de atendimentos clínicos do Hospital Veterinário Silvio Barbosa Cardoso (HVSBC) da Universidade Estadual do Ceará (UECE), no período de agosto de 2014 a junho de 2018, sem predileção de raça, peso ou idade. Como critério de inclusão no trabalho, as amostras de sangue deveriam apresentar positividade para a presença de microfilárias de Dirofilaria spp. em sangue periférico, observadas no esfregaço sanguíneo.

Em relação ao método de coleta do material sanguíneo, as amostras foram obtidas por punção venosa, utilizando-se tubos plásticos de coleta à vácuo (Vacutainer) e agulhas descartáveis de 0,7 x $25 \mathrm{~mm}$, com subsequente homogeneização manual do tubo. Após a coleta, as amostras foram processadas de acordo com a avaliação hematológica padrão, além de realização de perfil bioquímico.

Os dados hematológicos foram processados utilizando o analisador automatizado (BC 2800 Vet - Mindray®) obtendo-se valores referentes a eritrograma, plaquetograma e contagem total de leucócitos. A dosagem das proteínas plasmáticas totais e avaliação do plasma foi realizada utilizando refratômetro. A validação dos dados, bem como o diferencial leucocitário e a pesquisa de hemoparasitas foram realizados a partir de esfregaços sanguíneos corados pelo método panótico rápido. Os valores hematológicos foram comparados com os intervalos de referência descritos por Weiss e Wardrop (2010).

A análise do perfil bioquímico consistiu nos marcadores creatinina, albumina, fosfatase alcalina (FA), ureia e alanina aminotransferase (ALT), processados em aparelho de automação bioquímico veterinário (Labmax Plus®). Os valores de referências para os parâmetros citados foram de acordo com o descrito por Kaneko (2008). Todos esses dados foram então organizados em tabelas, para uma análise quantitativa com o suporte metodológico de Koche (2018), onde as alterações hematológicas e bioquímicas encontradas foram comparadas com o total de amostras positivas para Dirofilaria spp. consideradas no estudo, tanto em valores absolutos quanto em relativos. 


\section{Resultados e Discussão}

Em 2.400 amostras de sangue de cães analisadas, 26 apresentaram microfilárias $(1,1 \%)$ (Gráfico 1). Em uma pesquisa epidemiológica realizada em Cuiabá, Mato Grosso, de 633 amostras sanguíneas de cães, 3 (0,47\%) apresentaram-se positivas. (Fernandes et al., 2000) Em Porto Seguro, Bahia, em um trabalho realizado por Calazans (2018), de 45 amostras de sangue de cães, em $30(66,7 \%)$ foi possível visualizar as microfilárias.

Ressalta-se que, embora exista a possibilidade de visualização das microfilárias em amostras sanguíneas, há uma dificuldade de detecção destas quando seu número é inferior a 50 - 100 por mililitro (Nelson \& Couto, 2010). Fragmentos de microfilárias já foram encontradas, em outros estudos, em capilares glomerulares e peritubulares, onde achados histológicos destes animais foram consistentes com doenças crônicas, glomerulonefrite membranoproliferativa grave, observando ainda microfilárias intralesionais (Mircean et al., 2017).

Gráfico 1. Amostras hematológicas, evidenciando a porcentagem de amostras totais e percentual de amostras com presença de microfilárias.

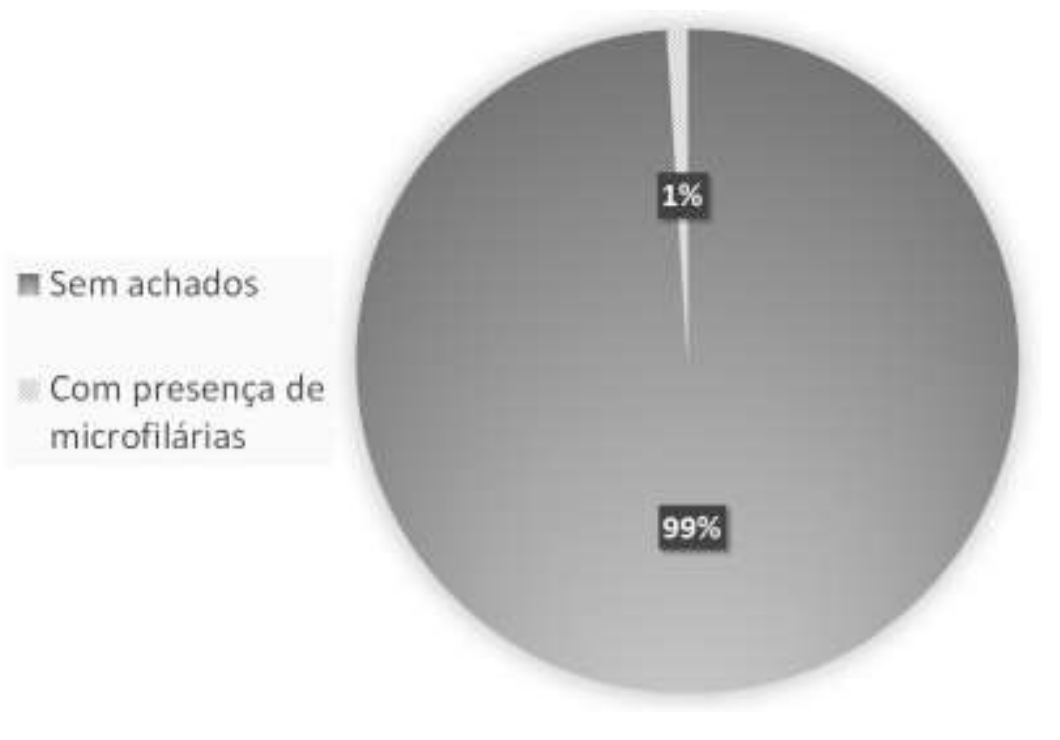

Fonte: Autores.

Nesse estudo, 46\% dos animais avaliados apresentaram sinais de regeneração eritrocitária (Gráfico 2), um indicativo de que a dirofilariose pode causar a destruição das hemácias e a perda dos elementos presentes na mesma. A anemia é um informativo para doença de dirofilariose grave, sendo a anemia mais severa em cães com síndrome da veia cava e doença pulmonar arterial grave. No caso da doença mais branda, as análises bioquímicas costumam ser normais com anemia regenerativa discreta (Salgueiro, 2016).

A anemia arregenerativa, por sua vez, é causada por lesões na medula óssea ou ausência de elementos necessários para a produção de eritrócitos (Freitas et al., 2018). Este tipo de anemia apresenta curso clínico crônico e início lento, é acompanhada de neutropenia e trombocitopenia (Silva \& González, 2008). 
Gráfico 2. Alterações hematológicas, evidenciando a porcentagem de amostras com presença de anemia e de amostras dentro da normalidade.

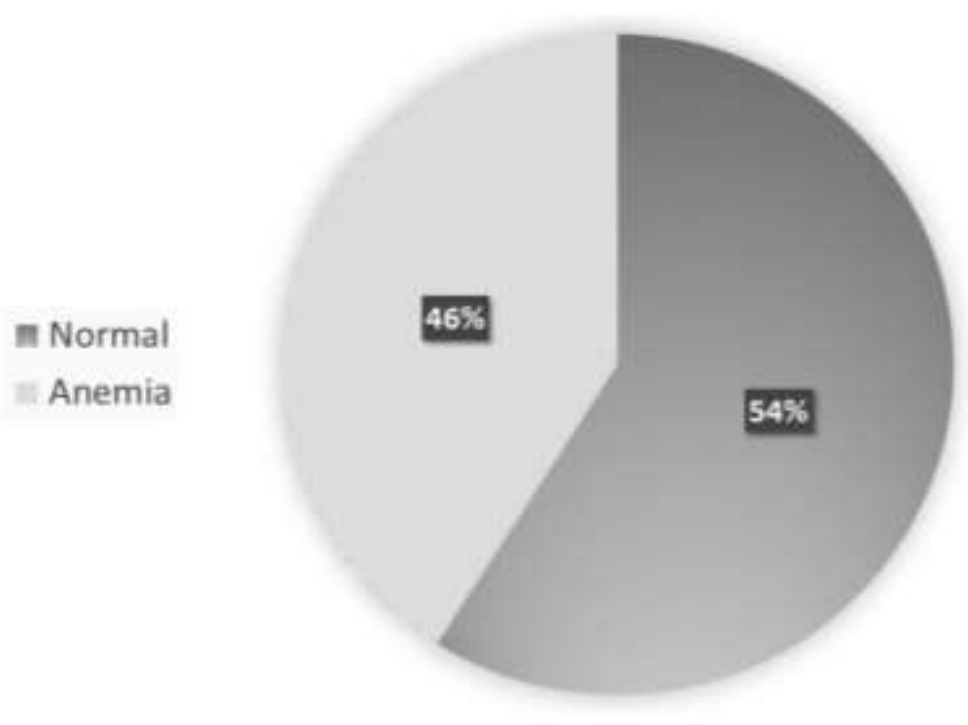

Fonte: Autores.

Nas alterações plaquetárias foi observado que entre 2.400 amostras, $42 \%$ apresentaram trombocitopenia (Gráfico 3). De acordo com Almosny (2002), quando presentes, as alterações mais comumente achadas em hemogramas são: anemia regenerativa, eosinofilia, basofilia, leucocitose com neutrofilia e trombocitopenia associados à tromboembolia. Wysmolek (2020) relatou que a trombocitopenia e anemia concomitante com babesiose em cães foram agravados em indivíduos coinfectados com D. repens. Conforme Jericó et al. (2015), a trombocitopenia é resultante da migração das plaquetas para o tecido pulmonar, onde ficam aderidas à parede dos vasos pulmonares. De acordo com Nelson e Couto (2010) a trombocitopenia pode ser resultado do consumo plaquetário pelo sistema arterial pulmonar.

Gráfico 3. Alterações leucocitárias, evidenciando a porcentagem de amostras com presença de trombocitopenia e de amostras dentro da normalidade (Média \pm DP).

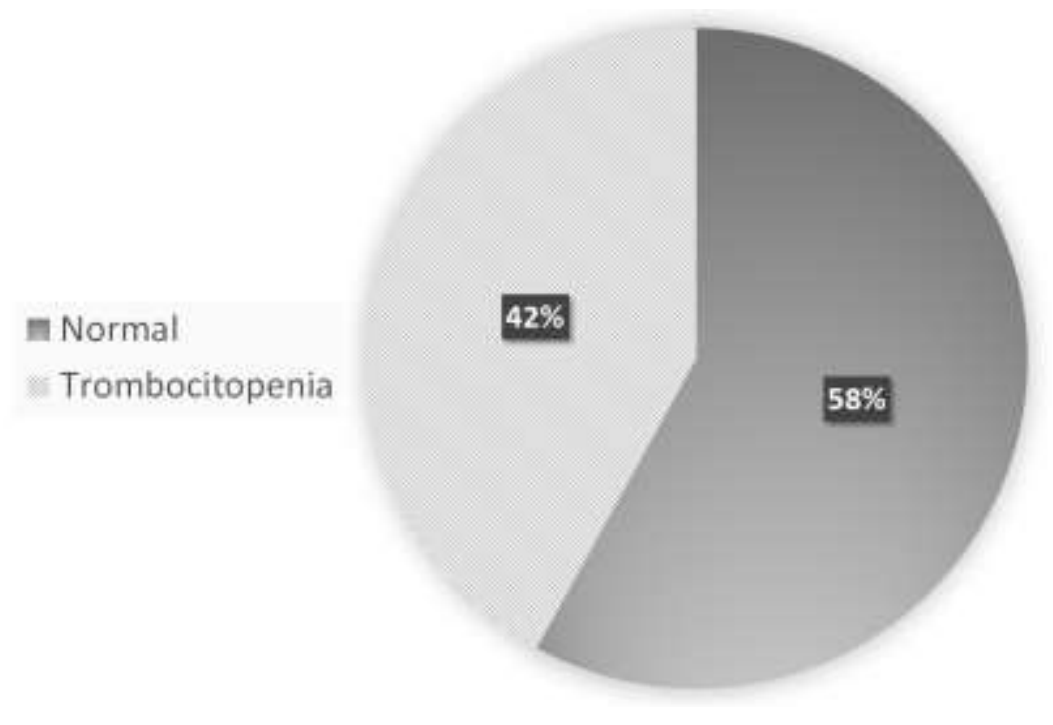

Fonte: Autores. 
Na série branca do hemograma, foi possível observar linfopenia em 1.464 pacientes, correspondendo a $61 \%$ dos casos (Gráfico 4). De acordo com Nelson e Couto (2015) achados como eosinofilia, basofilia, neutrofilia e monocitose são alterações comuns no exame hematológico em animais acometidos com dirofilariose, o que não corrobora com Jericó et al. (2015), que cita apenas as alterações de eosinofilia e basofilia como achados sugestivos de dessa doença em cães. Nesse caso, os achados leucocitários se basearam na diminuição das células linfóides no sangue, que de acordo com Wysmolek (2020), essa alteração pode estar associada a eventos de estresse ou ligados a infecção por microorganismo, gerando à liberação de glicocorticóides endógenos, podendo resultar na morte de linfócitos por apoptose ou sequestro dessas células pelos órgãos linfóides, como o baço.

Gráfico 4. Alterações leucocitárias, evidenciando a porcentagem de amostras que apresentaram diminuição de linfócitos e de amostras dentro da normalidade (Média \pm DP).

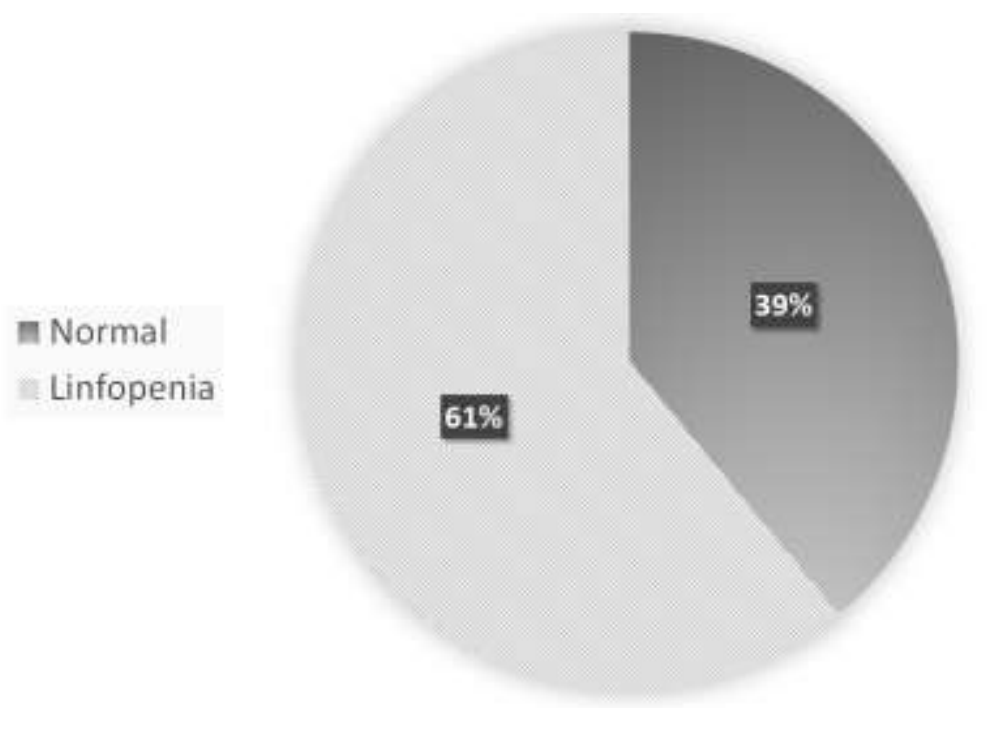

Fonte: Autores.

Neste trabalho, $41 \%$ dos cães estudados diagnosticados com dirofilariose apresentaram hiperproteinemia (Gráfico 5). Sabe-se que proteínas de fase aguda são proteínas plasmáticas, cuja concentração varia em resposta a traumas, infecções e à inflamação (Carretón et al., 2017). No caso de dirofilariose, é conhecido que essa enfermidade cause uma elevação na proteína c-reativa e na haptoglobina, principalmente em cães com alta carga parasitária, provavelmente devido à inflamação vascular causada pelas microfilárias e por suas bactérias endossimbiontes do gênero Walbachia (Méndez et al., 2015).

Carretón et al. (2017) relataram também que ambas as proteínas estão mais elevadas quando os cães apresentam hipertensão pulmonar associada a essa doença. A elevação das proteínas plasmáticas pode decorrer de alterações nas várias proteínas de fase aguda. Geralmente muitas delas devem estar elevadas para causar uma alteração eletroforética ou hiperglobulinemia. Porém, o fibrinogênio ou a haptoglobina podem estar isoladamente aumentados causando hiperglobulinemia e hiperproteinemia (Thrall et al., 2015). 
Gráfico 5. Análise proteica, evidenciando a porcentagem de amostras apresentando hiperproteinemia e de amostras dentro da normalidade (Média \pm DP).

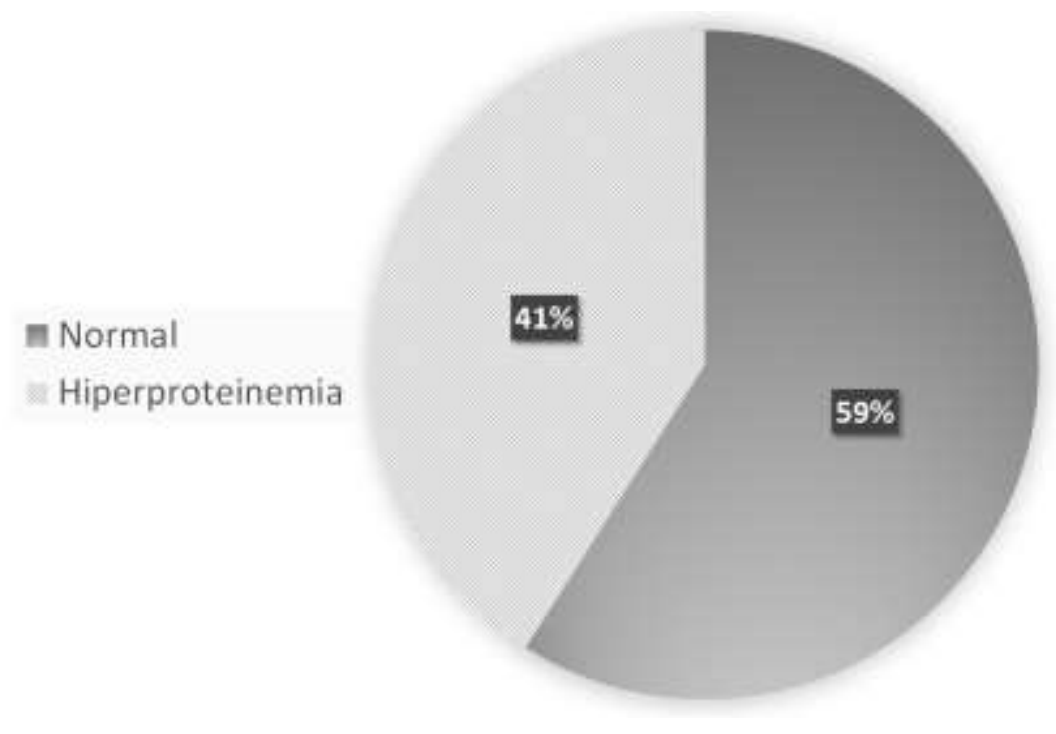

Fonte: Autores.

Em estudo de Carretón et al. (2020), 10,6\% dos cães acometidos de dirofilariose apresentaram hiperproteinemia e todos estes também tinham proteinúria. Assim como esse estudo, o proteinograma não foi realizado e a provável estimulação de globulinas relacionada à dirofilariose não foi avaliada detalhadamente. Sabe-se que há produção de anticorpos pelo hospedeiro contra muitas proteínas produzidas pela Dirofilaria, porém não são totalmente capazes de inativá-las (Simón et al., 2012). Em estudo de Milanović et al. (2017) foi encontrado um aumento significativo na fração de $\gamma$-globulina em cães acometidos de babesiose e dirofilariose, o que não ocorreu nos grupos do trabalho desses autores quando os animais só apresentavam babesiose ou nenhuma dessas doenças. Isso sugere que as imunoglobulinas como $\gamma$-globulina possam estar aumentadas no caso dos animais com hiperproteinemia do presente estudo.

No presente trabalho, $62 \%$ dos cães estudados diagnosticados com dirofilariose apresentaram hipoalbuminemia, mas não houve alterações nos exames laboratoriais hepáticos (Gráfico 6). De acordo com Nelson e Couto (2010), a hipoalbuminemia pode estar presente nos casos mais avançados podendo estar relacionada com a resposta imune do animal, visto que, como dito anteriormente, a elevação das proteínas plasmáticas pode decorrer de alterações nas várias proteínas de fase aguda, resultando no aumento das frações de $\beta$ e $\gamma$ globulinas, e a consequente queda da albumina, uma proteína de fase negativa da inflamação. Pode-se sugerir, dessa forma, que a hipoalbuminemia observada ocorreu devido ao estímulo inflamatório gerado. 
Gráfico 6. Presença de hipoalbuminemia, evidenciando a porcentagem de amostras que apresentaram hipoalbuminemia e de amostras dentro da normalidade (Média \pm DP).

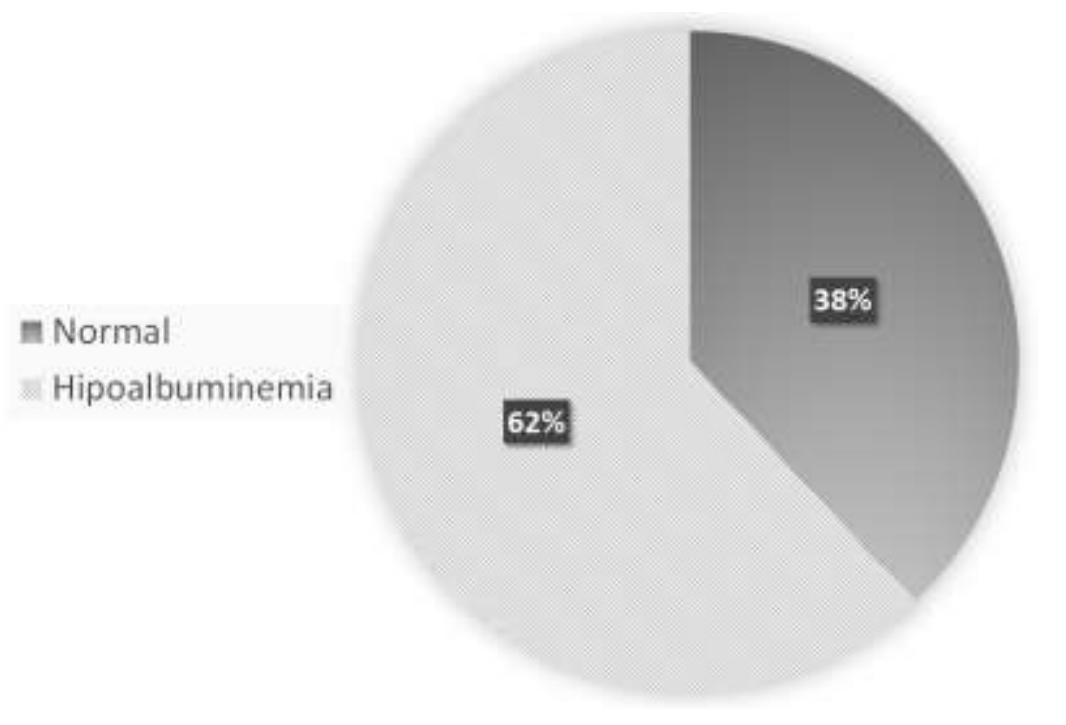

Fonte: Autores.

\section{Conclusão}

A dirofilariose tem maior ocorrência em cães, sendo que a maioria não apresenta sinais clínicos, contudo são considerados os reservatórios da doença. Foi relatado que a D. immitis pode desencadear anemia hemolítica devido à destruição das hemácias pela carga parasitária e, consequentemente, levando a regeneração eritrocitária.

Neste estudo também houve a apresentação de hiperproteinemia, sendo que nos casos de dirofilariose ocorre principalmente em cães altamente parasitados. Além disso, nos achados leucocitários mostrou-se a diminuição das células linfóides no sangue, deste modo, supondo uma infecção por microrganismos. Em relação ao perfil bioquímico é importante relatar que foi evidenciado albumina reduzida, sendo comumente observada em casos mais avançados da enfermidade e relacionada a resposta imunológica do animal.

Novas pesquisas a respeito dos aspectos epidemiológicos da dirofilariose no estado do Ceará podem ser realizadas, para uma maior elucidação das formas de prevenção, diagnóstico e tratamento dessa zoonose.

\section{Referências}

Almosny, N. R. P. (2002). Hemoparasitoses em pequenos animais domésticos e como zoonoses (1 ${ }^{\text {a }}$ ed.). L. F. Livros de Veterinária.

American Heartworm Society. (2014). Guideline for prevention, diagnosis, and management of heartworm (Dirofilaria immits) infection in Dogs.

Atkins, C. (2017). Canine Heartworm Disease. In S. J. Ettinger \& E. C. Feldman (Eds.), Textbook of Veterinary Internal Medicine (8th ed.) (pp. 3166-3228). Sauders.

Calazans, A. N. F. (2018). Epidemiologia da dirofilariose canina no município de porto seguro-bahia. [Dissertação de Mestrado em Ciência Animal, Universidade Estadual de Santa Cruz]. Biblioteca Virtual em Saúde. https://pesquisa.bvsalud.org/portal/resource/pt/vtt-217649

Carretón, E., Cerón, J. J., Martínez-Subiela, S., Tvarijonaviciute, A., Caro-Vadillo, A., \& Montoya-Alonso, J. A. (2017). Acute phase proteins and markers of oxidative stress to assess the severity of the pulmonary hypertension in heartworm-infected dogs. Parasites \& vectors, 10 (Suppl 2), 477. https://doi.org/10.1186/s13071-017-2426-8

Carretón, E., Falcón-Cordón, Y., Rodon, J., Matos, J. I., Morchón, R., \& Montoya-Alonso, J. A. (2020). Evaluation of serum biomarkers and proteinuria for the early detection of renal damage in dogs with heartworm (Dirofilaria immitis). Veterinary parasitology, 283 , 109144. https://doi.org/10.1016/j.vetpar.2020.109144

Dantas-torres, F., \& Otranto, D. (2020). Overview on Dirofilaria immitis in the Americas, with notes on other filarial worms infecting dogs. Veterinary Parasitology, v. 282, 109-113. 
Fernandes, C. G. N., Rodrigues, S., Moura, S. T., \& Oliveira, R. M. F. (2000). Aspectos Epidemiológicos da dirofilariose canina no perímetro urbano de Cuiabá, Mato Grosso: emprego do "Immunoblot" e do teste de Knot modificado. Braz. J. Vet. Res. Anim. Sci., 37(6).

Figueredo, L. A, Sales, K. G. D. S., Deuster, K., Pollmeier, M., Otranto, D., \& Dantas-Torres F. (2017). Exposure to vector-borne pathogens in privately owned dogs living in different socioeconomic settings in Brazil. Vet Parasitol., 243, 18-23. 10.1016/j.vetpar.2017.05.020.

Freitas, F. V., Siqueira, V. C. F., Vidal, N. B. C., Silva, R. O., Viana, I. L., Borges Filho, J. A., \& Leite, A. K. R. M. (2018). Dirofilarose em uma Cadela: Relato de caso. Revista Científica de Medicina Veterinária, 30(30), 327-336.

Furtado A. P., Carmo E. S., Giese E. G., Vallinoto A. C. R., Lanfredi R. M., \& Santos J. N. (2009). Detection of dog filariasis in Marajo Island, Brazil by classical and molecular methods. Parasitology Research, 105, 1509-1515. https://doi.org/10.1007/s00436-009-1584-9

Gonzalez, F. H. D., \& Silva, S. C. (Eds.). (2008). Patologia clínica veterinária: texto introdutório. Universidade Federal do Rio Grande do Sul.

Hodges, S., \& Rishniw, M. (2008). Intraarticular Dirofilaria immitis microfilariae in two dogs. Veterinary parasitology, 152(1-2), 167-170. https://doi.org/10.1016/j.vetpar.2007.11.018

Jericó, M. M., Neto, J. P. A., \& Kogika, M. M. (2015). Tratado de medicina interna de cães e gatos (1ª ed.). Roca.

Kaneko, J. J. (2008). Clinical Biochemistry of Domestic Animals (6 ${ }^{\circ}$ ed.). Academic Press.

Koche, J. $\quad$ C. (2011). Fundamentos de metodologia científica. Petrópolis: Vozes. https://www.google.com/url?sa=t\&rct=j\&q=\&esrc=s\&source=web\&cd=\&cad=rja\&uact=8\&ved=2ahUKEwjqz5DFurXxAhUNHbkGHYsMBnIQFjABegQIA xAD\&url=http\%3A\%2F\%2Fwww.adm.ufrpe.br\%2Fsites\%2Fww4.deinfo.ufrpe.br\%2Ffiles\%2FFundamentos_de_Metodologia_Cienti\%25CC\%2581fica.pdf \&usg=AOvVaw3xiVYbXfMLLroJgqqu4uhh.

Labarthe, N., Paiva, J., Reifur, L., Mendes-de-almeida, F., Merlo, A., Jose, C., Pinto, C., Juliani, P., Angela, M., Almeida, O., \& De Alves, L. C. (2014). Updated canine infection rates for Dirofilaria immitis in areas of Brazil previously identified as having a high incidence of heartworm-infected dogs. Parasites \& Vectors, 7,1-8.

Madril, A. B., da Silva, E. G., Alves, C. C., de Vasconcellos, A. L., de Sousa, E. P., \& Costa, P. P. C. (2020). Perfil hematológico de cães infectados por Dirofilaria immitis , 12., 2020, UNIPAMPA. Perfil hematológico de cães infectados por Dirofilaria immitis. Anais do Salão Internacional de Ensino, Pesquisa e Extensão, 12(2)

Magnis, J., Lorentz, S., Guardone, L., Grimm, F., Magi, M., Naucke, T. J., \& Deplazes, P. (2013). Morphometric analyses of canine blood microfilariae isolated by the Knott's test enables Dirofilaria immitis and D. repens species-specific and Acanthocheilonema (syn. Dipetalonema) genus-specific diagnosis. Parasites \& vectors, 6(48). https://doi.org/10.1186/1756-3305-6-48

Méndez, J. C., Carretón, E., Martínez-Subiela, S., Tvarijonaviciute, A., Cerón, J. J., \& Montoya-Alonso, J. A. (2015). Acute phase protein response in heartworm-infected dogs after adulticide treatment. Veterinary parasitology, 209 (3-4), 197-201. https://doi.org/10.1016/j.vetpar.2015.02.036

Milanović, Z., Ilić, A., Andrić, J. F., Radonjić, V., Beletić, A., \& Filipović, M. K. (2017). Acute-phase response in Babesia canis and Dirofilaria immitis coinfections in dogs. Ticks and tick-borne diseases, 8 (6), 907-914. https://doi.org/10.1016/j.ttbdis.2017.07.009

Mircean, M., Ionică, A. M., Mircean, V., Györke, A., Codea, A. R., Tăbăran, F. A., Taulescu, M., \& Dumitrache, M. O. (2017). Clinical and pathological effects of Dirofilaria repens and Dirofilaria immitis in a dog with a natural co-infection. Parasitology international, 66(3), 331-334. https://doi.org/10.1016/j.parint.2017.02.003

Moreira, H. R., Madeira, E. A. O., Cunha, D. N. L., Scofield, A., Góes-Cavalcante, G., Abel, I., Guimarães, R. J. P. S., \& Fernandes, J. I. (2019). Dirofilaria immitis infection in dogs in Algodoal Island, Brazilian Amazon. Pesquisa Veterinária Brasileira, 39(7), 510-515. https://doi.org/10.1590/1678-6160-PVB5916

Nelson, R. W., \& Couto, C. G. (2006). Fundamentos da medicina interna de pequenos animais (2 $\left.{ }^{\mathrm{a}} \mathrm{ed}.\right)$. Guanabara Koogan.

Nelson, R. W., \& Couto, C. G. (2010) Medicina interna de pequenos animais (4ª ed). Elsevier.

Nelson, R. W., \& Couto, C. G. (2015). Medicina interna de pequenos animais ( $5^{\mathrm{a}}$ ed.). Elsevier.

Otranto, D., Dantas-Torres, F., Brianti, E., Traversa, D., Petrić, D., Genchi, C., \& Capelli, G. (2013). Vector-borne helminths of dogs and humans in Europe. Parasites \& vectors, 6(16). https://doi.org/10.1186/1756-3305-6-16

Rebar, A. H., Lewis, H. B., DeNicola, D. B., Halliwell, W. H., \& Boon, G. D. (1981). Red cell fragmentation in the dog: an editorial review. Veterinary pathology, 18(4), 415-426. https://doi.org/10.1177/030098588101800401

Salgueiro, J. M. (2016). Dirofilariose Canina. (Master's thesis, Universidade Lusófona de Humanidades e Tecnologias, Faculdade de Medicina Veterinária).

Silva, J. D. S. M. D. (2018). Caracterização clínica e epidemiológica da dirofilariose cardiopulmonar canina no concelho de Benavente, Portugal (Doctoral dissertation, Universidade de Lisboa, Faculdade de Medicina Veterinária).

Smith, F. W., Tilley, L. P., Oyama, M., \& Sleeper, M. M. (2015). Manual of canine and feline cardiology (5 ed). Elsevier Health Sciences.

Silva, R. C., \& Langoni, H. (2009). Dirofilariose: Zoonose emergente negligenciada. Ciência Rural, 39(5), 1614-1623.

Simón, F., Siles-Lucas, M., Morchón, R., González-Miguel, J., Mellado, I., Carretón, E., \& Montoya-Alonso, J. A. (2012). Human and animal dirofilariasis: the emergence of a zoonotic mosaic. Clinical microbiology reviews, 25 (3), 507-544. https://doi.org/10.1128/CMR.00012-12

Thrall, M. A., Weiser, G., Allison, R. W., W., \& Campbell, T. W. (2015). Hematologia e Bioquímica Clínica Veterinária (2ª ed.). Roca. 
Research, Society and Development, v. 10, n. 8, e23010817252, 2021

(CC BY 4.0) | ISSN 2525-3409 | DOI: http://dx.doi.org/10.33448/rsd-v10i8.17252

Vieira, A. L., Vieira, M. J., Oliveira, J. M., Simões, A. R., Diez-baños, P., \& Gestal, J. (2014). Prevalence of canine heartworm (Dirofilaria immitis) disease in dogs of central Portugal. Parasite, 21, 5.

Wang, S., Zhang, N., Zhang, Z., Wang, D., Yao, Z., Zhang, H., Ma, J., Zheng, B., Ren, H., \& Liu, S. (2016). Prevalence of Dirofilaria immitis infection in dogs in Henan province, central China. Parasite, 23(43), 20-22.

Weiss, D. J., \& Wardrop, O. W. (2010). Schalm's Veterinary Hematology (6º ed.). Wiley-Blackwell.

Wysmołek, M. E., Dobrzyński, A., Długosz, E., Czopowicz, M., Wiśniewski, M., Jurka, P., \& Klockiewicz, M. (2020). Hematological and Biochemical Changes in Dogs Naturally Infected With Dirofilaria repens. Frontiers in veterinary science, 7(590). https://doi.org/10.3389/fvets.2020.00590 